\title{
Audit
}

\section{The importance of a safety checklist and strategic rest breaks in gynaecological laparoscopy}

\author{
D Silva ${ }^{a}$, P J Randombage ${ }^{b^{*}}$, W Gankanda ${ }^{b}$, J Mathanamohan ${ }^{c}$, M Rajakaruna ${ }^{b}$
}

\begin{abstract}
This study focuses on the importance of a safety checklist for gynaecological laparoscopic surgeries. Although several general safety checklists are used in practice (e.g.: The WHO Safety Checklist), there is no dedicated safety checklist for gynaecological laparoscopic surgeries.

Our aim is to introduce a safety checklist dedicated to gynaecological laparoscopy. It is based on our experience in performing various gynaecological laparoscopic surgeries in a tertiary care center with a substantially high workload. It has been compiled after studying the complications occurring in areas covered by the aforementioned checklist. We present data from 776 cases performed over 4 years at the Professorial Unit in Obstetrics and Gynaecology affiliated to the University of Sri Jayewardenepura, at Colombo South Teaching Hospital, Kalubowila, Sri Lanka. The mean surgical time and complications associated with patient positioning have been assessed.

Complications associated with the areas assessed were found to be of low incidence. However, we believe that these can be reduced further by the introduction of a checklist specifically tailored for gynaecological laparoscopy.
\end{abstract}

Key words: safety checklist, rest breaks, gynaecological laparoscopy, gynaecological endoscopy

\section{Introduction}

Gynaecological laparoscopy has gained popularity and been revolutionized in recent times. It offers a wide range of benefits such as minimal blood loss, early return to work, fewer requirements of post-operative analgesia and cosmetic advantages to name a few ${ }^{1}$. However, in more complex and extended surgeries, these advantages are undermined by the longer duration of time taken to perform them laparoscopically. This

Sri Lanka Journal of Obstetrics and Gynaecology 2021; 43: 326-333

DOI: http://doi.org/10.4038/sljog.v43i4.7989

a Senior Lecturer, Department of Obstetrics and Gynaecology, Faculty of Medical Sciences, University of Sri Jayewardenepura, Sri Lanka.

b Senior Registrar in Obstetrics and Gynaecology, Colombo South Teaching Hospital, Kalubowila, Sri Lanka.

c House Officer in Obstetrics and Gynaecology, Colombo South Teaching Hospital, Kalubowila, Sri Lanka.

Correspondence: PJR, e-mail: prabathrandombage@gmail.com

(iD https://orcid.org/0000-0002-3576-9923

Received $4^{\text {th }}$ April 2021

Accepted $12^{\text {th }}$ October 2021 
is related to the patient being in a non-physiological position for a longer duration of time, prolonged anesthesia and other complications such as deep vein thrombosis ${ }^{2}$.

Almost all gynaecological laparoscopies are conducted in a special steep Trendelenburg position, also known as the Lloyd Davies position. The position itself has its own complications such as lower limb compartment syndrome and hence has evolved over time into the "Modified Lloyd Davies" position to overcome these problems. However, various position related injuries are still encountered ${ }^{3}$.

The overall risk of major complications from laparoscopy is approximately 2 in 1000 cases. These risks need to explain to the patient prior to surgery, and an informed written consent that entails serious complications including damage to the bowel, bladder, ureters, uterus, major blood vessels, failure to gain laparoscopic entry, port site hernia, thrombo-embolic disease and death, should be taken from the patient as recommended by the Royal College of Obstetricians and Gynaecologists (RCOG) ${ }^{4}$.

Familiarity with the physiological changes associated with laparoscopic surgery is essential for appropriate patient selection and pre-operative assessment and helps to be equipped for any foreshadowed adverse events during surgery. This not only allows the surgeon to pre-plan the surgery, but also emphasizes on the importance of the involvement of a Multi-Disciplinary Team.

A pneumoperitoneum is achieved following insufflation of Carbon dioxide into the peritoneal cavity. The consequent rise in intra-abdominal pressure in turn leads to certain risks to the cardiovascular and respiratory systems. The raised intra-abdominal pressure pushes the diaphragm above, resulting in a reduced total lung volume by $35-40 \%$. In addition, the carbon dioxide absorbed through the peritoneal cavity causes hypercapnia that predisposes the patient to cardiac arrhythmias, and acidosis. Compression of renal vessels by the pneumoperitoneum can give rise to perioperative oliguria. Pressure on the inferior vena cava can reduce the pre-load to the heart leading to reduced cardiac output and low blood pressure, which however, is partly counteracted by the increase in venous return brought about by the Trendelenburg position. Nevertheless, this position also results in increased intra- cranial and intra-ocular pressures and reduced lower limb blood supply. Therefore, a proper understanding of the above changes allows the surgeon to predict complications and mitigate them accordingly with adequate planning, and implement strategic rest breaks corresponding to the complexity of the surgery.

Common position related injuries include but are not limited to rhabdomyolysis, nerve injuries which may be transient neuropraxia or rarely permanent, red eye, backache and headache especially at the occiput. Among them, nerve injuries account for the vast majority of cases due to prolonged surgical time.

The ulnar nerve is frequently impinged when the arm is in a pronated position with the patient in supine position. In addition, brachial plexus injuries can occur due to shoulder braces. Injury to the common peroneal and saphenous nerves can also occur due to prolonged use of leg stirrups 5 .

A continuous and methodical approach to patient safety in carrying out complex and prolonged interventions is the cornerstone of risk management strategies in gynaecological laparoscopy ${ }^{6}$.

In order to meet the objectives of patient safety, the WHO has introduced a WHO safety checklist, which has proven effective in reducing surgical morbidity, mortality and improving communication and teamwork $^{7,8}$. However, this checklist is a rather general approach to all surgeries and not specific to laparoscopy or gynaecological laparoscopy.

Considering the special safety measures related to laparoscopy, some studies have proven that integrated operating rooms and implementing pre-operative online checklists are effective in reducing some of the intraoperative laparoscopy related complications. These were conducted in other surgical disciplines and were not specific to gynaecological procedures which have position related complications of their $\mathrm{own}^{9}$. In addition, some surgeons raise questions regarding its applicability on a wider context, as certain surgeries may require comprehensive checklists which are specific to the particular surgery being performed ${ }^{10}$.

Some studies focused on technical aspects and introduced structured checklists related to equipment ${ }^{11}$. Nevertheless, they failed to demonstrate efficacy in preventing complications related to advanced laparoscopic procedures. 
Prolonged surgical time is a common denominator to both laparoscopic and robotic surgeries. However, robotic surgeries have other characteristics as well; the position of the surgeon is more distant from the patient, obscuration of the patient by robotic arms and extended draping. Therefore, this demands frequent reviews of the patient. Having this in mind, safety checklists which are unique to robotic surgeries have been put forward and have been shown to be effective².

Gynaecological laparoscopy has seen rapid advancement in recent times. Prolonged surgeries involving the pelvis such as that for endometriosis are being performed effectively with the use of laparoscopy. Characteristics of these surgeries include patient positioning (the modified Lloyd Davies position), port placement towards the pelvis and safety measures to displace the bowel off from the surgical field.

However, taking existing evidence into account, there are no safety checklists specifically tailored for gynaecological laparoscopy. We conducted an audit to identify unique complications related to advanced gynaecological procedures. This manuscript is compiled with the purpose of introducing a safety checklist for such procedures by utilizing our expertise in performing advanced gynaecological laparoscopy spanning over a decade.

\section{Methods}

\section{Laparoscopic safety checklist}

Safety measures in laparoscopy focus on several aspects, the most important of them being the comprehensive pre-operative head to toe examination of the patient. (Figure 1)

The checklist begins at the head, where the correct placement of the head ring is confirmed. This ring is preferably made of a soft material, which provides a cushioning effect and therefore prevents position related injuries to the head. These injuries mainly include contusions over the occiput and post-operative headache.

Prolonged laparoscopic procedures lead to various complications related to the eyes, such as dry eyes, red eyes, conjunctival oedema and severe irritation, which can easily be prevented by applying eye pads. Soft gauze swabs secured using a plaster can be used in a low resource setting.
The next factor to be considered is the insertion of a naso-gastric tube ideally prior to endo-tracheal intubation, if a Palmer's point entry is decided upon due to past surgery. It prevents inadvertent entry into the stomach. Proper communication and co-ordination with the Anaesthetist are paramount as it is easier for the anesthetist to insert a naso-gastric tube prior to endo-tracheal intubation.

Arms should be safely placed alongside the patient's body and secured using specially made straps or simply by wrapping them with a folded sheet. In addition, the surgeon should ensure adequate spacing between the arms and the body and also be mindful of the arms being pressurized by metal parts of the theatre table to prevent contusions and necrosis of the fingers.

The next essential step would be to ensure proper placement of the shoulder guard. It is vital to see that it is not placed over the acromial bony prominences in order to prevent mechanical type of shoulder pain following a prolonged surgery. Ideally, they should be placed bilaterally, but if the resources are limited, it should be placed at the side of the assistant, allowing the surgeon to move more freely. Gel pads should be placed underneath the shoulder guards.

The surgeon should also ensure adequate spacing between the neck and the shoulder, which helps prevent neck pain as well as brachial plexus injuries.

Since most of the gynecologists opt to approach the patient from the left, the left arm of the patient should be placed safely alongside the body while the intravenous access is from a cannula placed on the right arm. This arm is placed on an arm rest and can be placed at a comfortable anatomically correct angle to the patient's body.

As demonstrated in Figure 2, the arm should be bent slightly to facilitate anatomically correct positioning and the assistant stands behind the arm rest during surgery (Figure 2).

Subsequently, both of the legs should be placed on pneumatic adjustable stirrups. Where these are unavailable and when conventional leg rests are used, the lower side of the knees and ankles should be protected by gel pads. Pneumatic compression stockings should be used and applied appropriately prior to commencement of prolonged surgery in order to prevent deep vein thrombosis. 


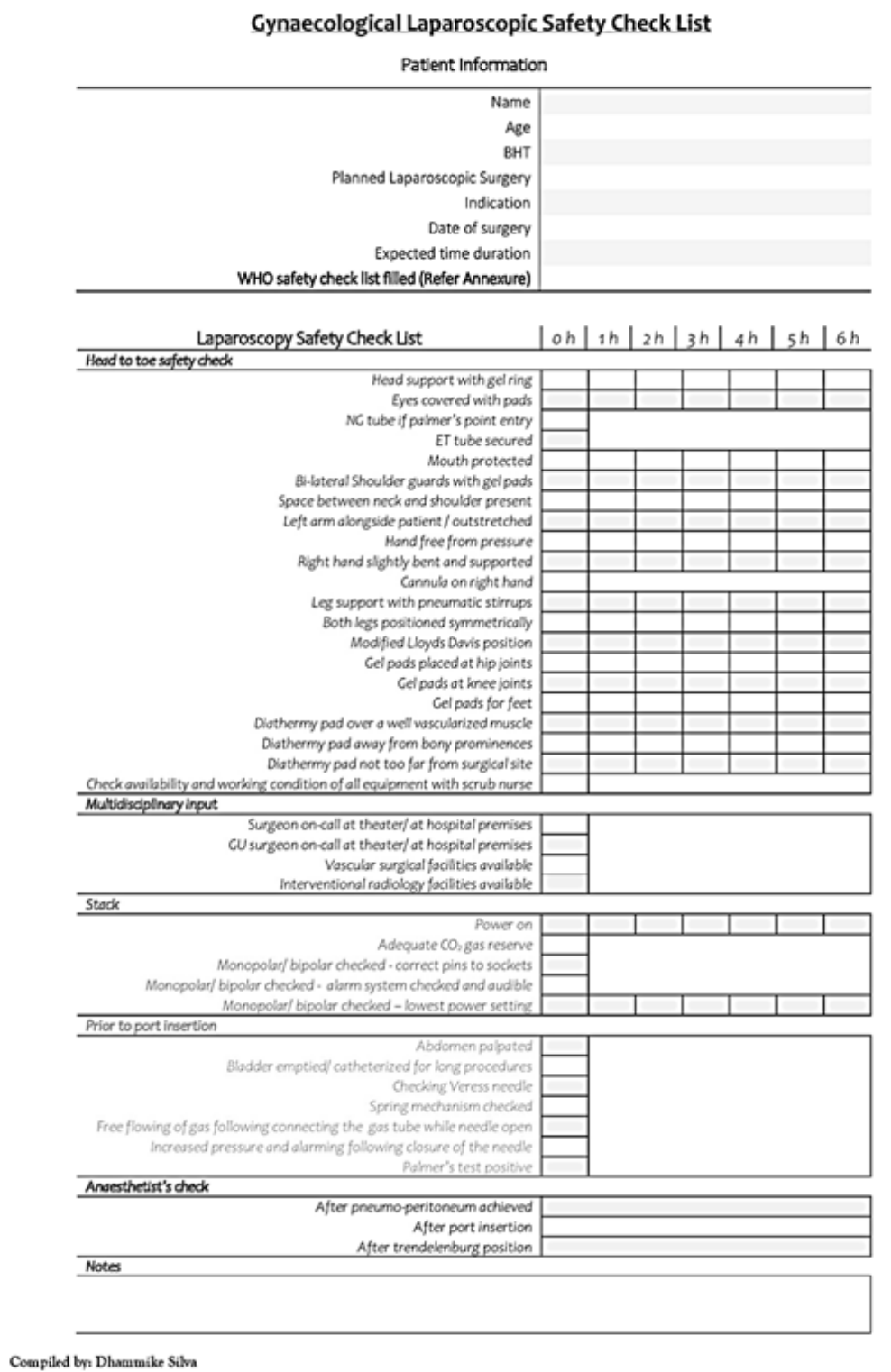

Figure 1. Gynaecological laparoscopic safety checklist.

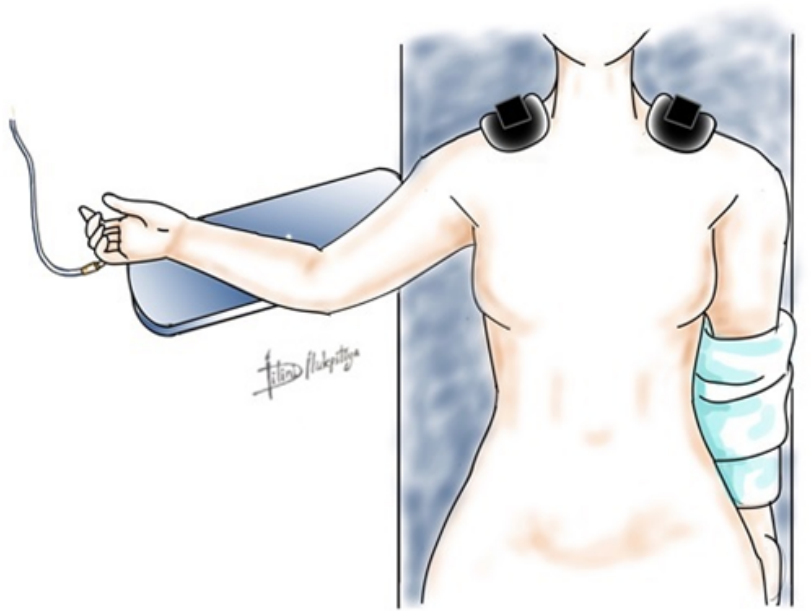

Figure 2. Positioning of arms and shoulder guards. 
Diathermy pads should be placed properly to prevent burn injuries. It should be placed over a highly vascular muscle mass away from bony prominences. Return electrode monitoring system of the pads will detect any form of detachment as a result of an increase in impedance in the attachment area. In gynaecological laparoscopy, the most convenient site is the upper thigh.

Bladder catheterization or emptying of the bladder prior to abdominal entry also account for safety measures by reducing the likelihood of entry related bladder injuries. Avoiding suprapubic veress insertion and inserting all secondary trocars under direct vision are additional measures that can avoid urinary tract injuries. Furthermore, minimizing the use and spread of electrosurgery will reduce the risk of thermal damage.

It is essential to ensure the availability of a multidisciplinary team prior to commencing advanced gynaecological laparoscopy. The team should essentially include a colorectal surgeon and a genito-urinary surgeon.

Finally, checking of the laparoscopic stack and instruments should also be made part of the safety checklist, and this has been addressed by other studies as well ${ }^{11}$.

\section{Primary survey of a clinical audit}

The purpose of this manuscript is to present the primary survey of a clinical audit that emphasizes on the introduction of a laparoscopic safety checklist, conducted at the Colombo South Teaching Hospital, Kalubowila, Sri Lanka. The study population of this audit comprises of all the laparoscopic surgeries conducted at the aforementioned hospital from January 2016 to January 2020. It is of importance to note that all the surgeries were conducted by one surgeon, which effectively excludes inter operator bias. A total of 776 patients were analysed and the data was gathered from an electronic database. The Mean surgical time and its Standard Deviation were analysed amongst the various types of surgical procedures, as it is the key determining factor in position related complications. A re-audit for this novel safety checklist is expected to be performed over the next 4 years from the time of implementation.

\section{Results}

Table 1. Distribution of the type of gynaecological laparoscopic surgeries and mean surgical time

\begin{tabular}{|l|l|c|}
\hline Type of gynaecological laparoscopic surgeries & N & Mean surgical time (SD) \\
\hline Surgeries for deep infiltrating endometriosis/ adhesiolysis & 42 & $190.80(41.68)$ \\
Ureteric re-implantation & 4 & $138.30(35.37)$ \\
Sacro-colpopexy & 22 & $148(36.23)$ \\
Burch colposuspension & 5 & $108.60(26.32)$ \\
Myomectomy and adenomyomectomy & 82 & $86.35(51.91)$ \\
Total laparoscopic hysterectomy & 288 & $60.8(27.26)$ \\
Cystectomy for endometrioma & 209 & $81.8(26.44)$ \\
Cystectomy for other types of adnexal cysts & 124 & $72.34(26.64)$ \\
\hline
\end{tabular}

Mean surgical time for deep infiltrating endometriosis and adhesiolysis was 190.80 (SD +/_ 41.68) minutes. The mean surgical time for other major surgeries such as for laparoscopic sacro-colpopexy was 148 (SD +/- 36.23), Burch colposuspension was 108.60 (SD +/- 26.32) and ureteric reimplantation was 138.30 (SD +/- 35.37). 
Table 2. Complications linked to areas addressed by the safety checklist

\begin{tabular}{|l|c|}
\hline Complication & N (percentage \%) \\
\hline Contusions over the occiput & $12(0.015)$ \\
Headache & $28(0.037)$ \\
Dry eyes & $16(0.021)$ \\
Red eyes, conjunctival oedema & $34(0.044)$ \\
Inadvertent entry into stomach & $2(0.002)$ \\
Contusions of fingers & $3(0.003)$ \\
Shoulder pain & $46(0.060)$ \\
Neck pain & $32(0.042)$ \\
Backache & $39(0.051)$ \\
Neuropraxia of nerves of upper limbs & $28(0.037)$ \\
Permanent nerve injuries of upper limbs & 0 \\
Neuropraxia of nerves of lower limbs & $14(0.018)$ \\
Permanent nerve injuries of lower limbs & 0 \\
Deep vein thrombosis & $22(0.029)$ \\
Diathermy burn injuries & $2(0.002)$ \\
Entry related bladder injuries & $6(0.007)$ \\
\hline
\end{tabular}

The commonest complication was found to be shoulder pain $\{n=46(0.060 \%)\}$, while the least common were diathermy injuries and entry related stomach injuries. $\{\mathrm{n}=2(0.002 \%)\}$.

There were no permanent nerve injuries in the upper and lower limbs.

\section{Discussion}

\section{Main findings}

Overall, complications related to the areas assessed in the gynaecological safety checklist were significantly low. Complications can occur due to the lack of safety measures, especially related to positioning and prolonged duration. We believe that these complications could be effectively mitigated by the implementation of a tailored Safety Checklist. This should be instigated during the pre-operative period as well as at regular intervals during the intra-operative period.

Laparoscopic surgery is more tiring in comparison to open surgery owing to the lack of depth perception and limited operative freedom of movements. It also requires the surgeon to stand still and focus on a screen in a dim-lit surrounding for prolonged durations. These in turn lead to muscle stiffness and eye strain. Regardless of the complexity or simplicity of a surgery and the skill level of the surgeon, fatigue can contribute to errors at the expense of the patient. Therefore, the authors believe that short breaks in between prolonged surgeries are beneficial to both the patient and the surgeon, as the patient can be repositioned back to the supine position from the steep Trendelenberg position which helps reduce position related complications and also gives an opportunity for the surgeon to tackle 
physical and mental exhaustion. Nevertheless, the implementation of such breaks would necessitate reevaluating the safety checklist while repositioning the patient again.

Setting up the operating theater ergonomically is of paramount importance and its implication cannot be overstated. A relaxed surgeon is allowed to focus on the intricacies of the surgery when not distracted by physical exhaustion. Therefore, appropriate placement of instruments corresponding to the height of the surgeon's elbow, and positioning the screens slightly below the level of the surgeon's eyes will enable the surgeon to work comfortably.

\section{Interpretation}

As mentioned above, the WHO Safety Checklist is a more general approach and neither specific to laparoscopic procedures nor gynaecological procedures in particular ${ }^{7,8}$. In addition, most of the safety checklists concentrate on surgical equipment and safety concerns that are specific to certain surgical steps ${ }^{10,11}$.

A similar study has been undertaken among robotic surgical procedures, which highlights the prolonged nature of these surgeries and specific key steps that need to be followed during robotic surgeries ${ }^{3}$. Our survey stands out from these as we pay more attention to position related injuries which can be easily prevented, and that are more specific to gynaecological laparoscopic safety.

\section{Strengths and limitations}

This clinical audit has been carried out in one of the leading centers of excellence in gynaecological laparoscopy in Sri Lanka, where a wide range of surgeries are being performed on a daily basis.

The most striking characteristic of this study is that all the surgeries were performed by one Consultant Gynaecologist which effectively nullifies inter-operator bias. According to currently existing evidence, there is no established safety checklist for gynaecological laparoscopy, and this would be the first of its kind.

However, the effectiveness of this Safety Checklist should be evaluated by completing this audit cycle having the feasibility, patient and surgical team satisfaction and convenience in mind.

\section{Conclusion}

Although, the incidence of complications related to the areas assessed were significantly low, we believe that these can be reduced further by implementing a safety checklist specifically designed for gynaecological laparoscopy.

\section{Author declarations}

Conflicts of interest: None.

Funding: None

Ethical approval: Not applicable.

\section{Acknowledgements}

We would like to show our gratitude to the Director and theatre staff of Operation theatre B, Colombo South Teaching Hospital, Kalubowila, Sri Lanka for their support in conducting this study. We would also like to appreciate Dr. Dillini Illukpitiya, Registrar in Obstetrics and Gynaecology and Dr. Vidura Edirisinghe, Relief House officer in Colombo South Teaching Hospital, Kalubowila, Sri Lanka for their illustrations.

\section{References}

1. Hayashi H, Ozaki N, Ogawa K, Ikuta Y, Tanaka H, Ogata $\mathrm{K}$, et al. Assessing the economic advantage of laparoscopic vs. open approaches for colorectal cancer by a propensity score matching analysis. Surg Today [Internet]. 2018; 48(4): 439-48. Available from: https://doi.org/10.1007/s00595017-1606-7

2. Song JB, Vemana G, Mobley JM, Bhayani SB. The second "time-out": a surgical safety checklist for lengthy robotic surgeries. Patient Saf Surg [Internet]. 2013; 7(1): 19. Available from: https:// doi.org/10.1186/1754-9493-7-19

3. Tomassetti C, Meuleman C, Vanacker B, D'Hooghe T. Lower limb compartment syndrome as a complication of laparoscopic laser surgery for severe endometriosis. Fertil Steril [Internet]. 2009; 92(6): 2038.e9-2038.e12. Available from: http:// www.sciencedirect.com/science/article/pii/ S0015028209034517

4. RCOG. Diagnostic Laparoscopy Consent Advice No.2. 2017; (2): 8. Available from: https:// www.rcog.org.uk/globalassets/docume 
5. Cheney FW, Domino KB, Caplan RA, Posner KL. Nerve injury associated with anesthesia A closed claims analysis. Anesthesiol J Am Soc Anesthesiol. 1999;90(4):1062-9.

6. Singh SS, Condous G, Lam A. Primer on risk management for the gynaecological laparoscopist. Best Pract Res Clin Obstet Gynaecol [Internet]. 2007 Aug 1 [cited 2019 Aug 15]; 21(4): 675-90. Available from: https://www.sciencedirect.com/ science/article/pii/S1521693407000363

7. Solsky I, Berry W, Edmondson L, Lagoo J, Baugh J, Blair A, et al. World Health Organization Surgical Safety Checklist Modification: Do Changes Emphasize Communication and Teamwork? J Surg Res. 2020; 246: 614-22.

8. Haugen AS, Sevdalis N, Sųfteland E. Impact of the World Health Organization Surgical Safety Checklist on Patient Safety. Anesthesiology. 2019; 131(2): 420-5.
9. Buzink SN, van Lier L, de Hingh IHJT, Jakimowicz JJ. Risk-sensitive events during laparoscopic cholecystectomy: the influence of the integrated operating room and a preoperative checklist tool. Surg Endosc [Internet]. 2010; 24(8): 1990-5. Available from: https://doi.org/10.1007/s00464010-0892-6

10. Connor SJ, Perry W, Nathanson L, Hugh TB, Hugh TJ. Using a standardized method for laparoscopic cholecystectomy to create a concept operation-specific checklist. HPB [Internet]. 2014 May 1 [cited 2019 Aug 15];16(5):422-9. Available from: https://www.sciencedirect.com/science/ article/pii/S1365182X15315793

11. Verdaasdonk EGG, Stassen LPS, Hoffmann WF, van der Elst M, Dankelman J. Can a structured checklist prevent problems with laparoscopic equipment? Surg Endosc [Internet]. 2008; 22(10): 2238-43. Available from: https://doi.org/10.1007/ s00464-008-0029-3 